\title{
Discrimination of Munitions and Explosives of Concern at F.E.Warren AFB using Linear Genetic Programming
}

\author{
Frank D. Francone \\ RML Technologies Inc. and Chalmers \\ Univ. of Technology \\ 7606 S. Newland St. \\ Littleton CO 80128 \\ $+1.720 .981 .8710$ \\ fdf@francone.com
}

\author{
Larry M. Deschaine \\ Science Applications Int. Corp and \\ Chalmers Univ. of Technology \\ 272 Parker Rd. \\ Edgefield SC 29824 \\ $+1.706 .951 .2750$ \\ Larry.M.Deschaine@SAIC.com
}

\author{
Jeffrey J. Warren \\ Science Applications International \\ Corp. \\ 6310 Allentown Boulevard \\ Harrisburg, PA 17112 \\ $+1.717 .901 .8828$
}

\begin{abstract}
Removing underground, unexploded bombs, mortars, cannonshells and other ordnance ("MEC" or "UXO") from former military ranges is difficult and expensive. The principal difficulty is discriminating intact, underground ordnance from other metallic items such as fragments of exploded ordnance ("Clutter"), magnetic rocks, and "historic" items such as horseshoes, barbed-wire, and refrigerators. This study represents the first, large-scale, blind-test of MEC discrimination technology on production-grade, survey-mode data from the cleanup of a real impact site. The results reported here significantly advance the state-of-the-art in MEC discrimination over alternative forwardmodeling/inversion approaches to performing MEC discrimination. We combined Linear Genetic Programming (LGP) and statistical analysis to process data from the cleanup of 600 acres of the F.E.Warren Air Force Base. These data contained almost 30,000 targets of interest identified by geophysicists, including three-hundred thirty-two $75 \mathrm{~mm}$ projectiles $(75 \mathrm{~mm})$ and $37 \mathrm{~mm}$ projectiles $(37 \mathrm{~mm})$. A little under one-third of the groundtruth was held back by the customer for blind-testing. Our task was to discriminate intact $37 \mathrm{~mm}$ 's and $75 \mathrm{~mm}$ 's from the clutter by ordering the targets from most-likely to be MEC to least-likely to be MEC in what is referred to as a "prioritized diglist". We identified all $75 \mathrm{~mm}$ 's by $28.2 \%$ of the way through our prioritized dig-list and all $37 \mathrm{~mm}$ 's by $64.2 \%$ of the way through the prioritized dig list. Thus, depending on ordnance type, we reduced the number of targets that had to be excavated (false alarms) to clear the entire site by between $35 \%$ and $72 \%$.
\end{abstract}

\section{Categories and Subject Descriptors}

I.2.2 [Artificial Intelligence]:Automatic Programming, Program Synthesis. J.2 [Computer Applications] Physical Sciences and Engineering.

Permission to make digital or hard copies of all or part of this work for personal or classroom use is granted without fee provided that copies are not made or distributed for profit or commercial advantage and that copies bear this notice and the full citation on the first page. To copy otherwise, or republish, to post on servers or to redistribute to lists, requires prior specific permission and/or a fee.

GECCO'07, July 7-11, 2007, London, England, United Kingdom. Copyright 2007 ACM 978-1-59593-697-4/07/0007...\$5.00.

\section{General Terms}

Algorithms, Measurement, Performance, Economics, Experimentation, Verification.

\section{Keywords}

Linear Genetic Programming; Discipulus; Geophysics; Unexploded Ordnance; UXO; Munitions and Explosives of Concern; MEC; EM61 MK2.

\section{INTRODUCTION}

Buried unexploded ordnance ("UXO") (or Munitions and Explosives of Concern ("MEC")) on closed military bases poses a hazard to life-and-limb and further prevents huge tracts of landfrequently urban - from being returned to civilian use. The main barrier to cleaning-up closed military bases is cost. The Department of Defense ("DoD") stated: "The UXO cleanup problem is a very large-scale undertaking involving 10 million acres of land at some 1400 sites."1 One of the key problems is, according to DoD, ". . . instruments that can detect the buried UXO's also detect numerous scrap metal objects and other artifacts, which leads to an enormous amount of expensive digging. Typically 100 holes may be dug before a real UXO is unearthed!'[6]. It costs more than $\$ 100$ to excavate each target location that might contain a MEC item. So most of the cost of base-cleanup is spent digging empty holes, horseshoes, magnetic rocks and "MEC Clutter" or "Clutter"- small pieces of ordnance that flew apart on detonation or impact and pose no explosive hazard.

This study represents the first, large-scale, blind test of MEC discrimination technology on production-grade, survey-mode data from the cleanup of a real impact site. The results reported here significantly advance the state-of-the-art in MEC discrimination over alternative forward-modeling/inversion approaches to the subject.

\subsection{Digital Geophysical Sensors}

Geophysicists locate possible MEC items on a site by pulling passive magnetic or active electromagnetic sensors across the site in parallel lines (also called "transects") spaced 0.5 to 1 meter apart. These data are plotted spatially and potential MEC items show up as anomalies in an otherwise reasonably flat signal field. This process is referred to as Digital Geophysical Mapping 
("DGM"). The anomalous regions in the DGM are referred to as targets. This paper describes a process for determining which Targets are MEC and which are harmless metallic objects.

In this project we used the signal from a four-channel, active electromagnetic sensor, the Geonics EM61 MK2. ${ }^{1}$ It records the magnetic signal induced in an underground object by an electromagnetic pulse at three time-decay intervals at a lower coil. The fourth channel is measured at a coil placed above the lower coil-the "upper coil."

MEC items range in mass from a few ounces to 2000 pounds. Almost all buried metallic clutter items on a site fall in the same weight-range (the vast bulk in the lower end of that range). So the task of discriminating between smaller MEC and Clutter cannot rely on mass - it has to identify objects with typical MEC characteristics. For example, most MEC is roughly cylindrical and has a length to diameter ratio of four or five to one. By way of contrast, magnetic rocks and metallic clutter are normally irregular and less elongated.

\subsection{Previous Work}

Two approaches to MEC discrimination have shown promise. In one approach, a parameterized forward-model derived from the physics of magnetometers and electromagnetic induction is derived. This approach uses theory to reduce all information about a given Target to the set of parameters (between 2 and 5 parameters) that drive the forward model. To discriminate a Target, the forward-model is inverted and the parameters that best fit the actual measured DGM of a Target are extracted. The parameters are then used in a variety of ways to classify the Target as MEC or not-MEC [3][4][9][15].

The advantage of the forward-modeling/inversion approach is that it works from known physics approximations of actively-induced magnetism and passive magnetometers. The principal shortcomings of this approach are: (1) It does not produce a unique solution for the parameters for a large number of targets; (2) For effective discrimination, it requires very accurate positional information ( $<=1 \mathrm{~cm}$ error), which is not attainable with current, economic positioning technology for sensors moving across the field ("survey" mode) [18]; and (3) It requires higher signal-to-noise ratios than are typically encountered in production data for smaller targets like $37 \mathrm{~mm}$ MEC.

Thus, forward modeling/inversion technology is mostly confined to "cued" data - that is, data collected at very high resolution and positional accuracy with static measurements over a known Target location or research grade survey data. Further, studies using the this approach have not reported much success in discriminating small ordnance $(20 \mathrm{~mm}$ and $37 \mathrm{~mm}$ projectiles) most of the successes have been with larger ordnance.

The other approach was pioneered by Deschaine in 2002. He applied Linear Genetic Programming ("LGP") to research-grade data from a Protem- 47 sensor and compared his results to those of the ten other contractors who had attempted discrimination on these same data. Deschaine, using LGP, did a dramatically better

\footnotetext{
1 For more information about the EM61 MK2, please see www.geonics.com.
}

job discriminating MEC from not-MEC than had been reported by the other ten contractors [7].

Later, Banks applied a Genetic Programming approach to these same data [1]. His preprocessing and Genetic Programming modality were different than Deschaine's. Nevertheless, he produced results significantly better than the other ten contractors; but not as good as Deschaine's.

Francone and Deschaine then applied LGP to high-quality, survey-mode, production-grade EM61 MK1 data from a DoD test bed. That test-bed attempted to simulate an actual MEC impactsite with known buried items. Francone [12][11] reported in that study that LGP reduced the number of false-alarms (the number of non-MEC items that had to be dug to completely clear the site of MEC items) by about $40 \%$. This was particularly significant as the smallest items on that site were $20 \mathrm{~mm}$ projectilestraditionally regarded as very difficult to discriminate from clutter. On the other hand, the sample size in that study was very small (a total of only 17 MEC items and 300 non-MEC targets). So some question remained whether the results would replicable on larger data sets.

The principal advantages of Francone and Deschaine's LGP approach to-date are: (1) It has demonstrated success on fieldgrade, simulated, production data gathered in survey-mode; and (2) It has demonstrated success in discriminating small ordnance from surrounding clutter [12].

This paper reports the results of applying an extension of the methodologies reported by Francone and Deschaine in [12] to a large, MEC cleanup project using only production-grade, surveymode data.

\section{THE WARREN AIR FORCE BASE DATA}

F.E.Warren Air Force Base ("Warren") is located near Cheyenne, Wyoming. In the past, a portion of that base served as a practice range, primarily for $75 \mathrm{~mm}$ and $37 \mathrm{~mm}$ projectiles.

We analyzed DGM data comprising over 60 million data points in four channels of data from 600 acres of Warren (the "Site"). Each data point consisted of four channels of information gathered from a Geonics EM61 MK2 configured with three time-decay channels on the lower-coil and one upper-coil channel. These data were integrated with a differential global positioning system (GPS). They were collected with one-meter between transects. ${ }^{2}$

We were also provided a table containing geophysicist-designated target-locations on the Site (the "Targets") and ground-truth for some of those Targets (groundtruth is what the dig-teams actually found when they dug that Target up). Altogether, there were 29,130 Targets designated. The vast bulk of them contained one or more metallic items on excavation.

\footnotetext{
2 The DGM of the Site was delivered to us already lag-corrected by the customer in the Geosoft Oasis-Montaj UXO processing module. Lag correction is the process of adjusting the $\mathrm{x}, \mathrm{y}$ coordinates of each data point to reflect the fact that alternate lines of data are collected when the sensors are traveling different directions. We assumed, for the purpose of this study that no further lag correction was necessary or desirable.
} 
Of these 29,130 Targets, only 332 contained $37 \mathrm{~mm}$ or $75 \mathrm{~mm}$ MEC items. The remainder contained mostly MEC Clutter.

\section{TARGET DENSITY}

The Target density across the 600 acre Site varied widely. Some areas were densely populated with buried metal. Figure 1 shows the Targets-per-acre on the Site sampled in 20x20 meter squares. The density ranges from low to over 400 Targets-per-acre.

Figure 1. Target Density (Targets per Acre)

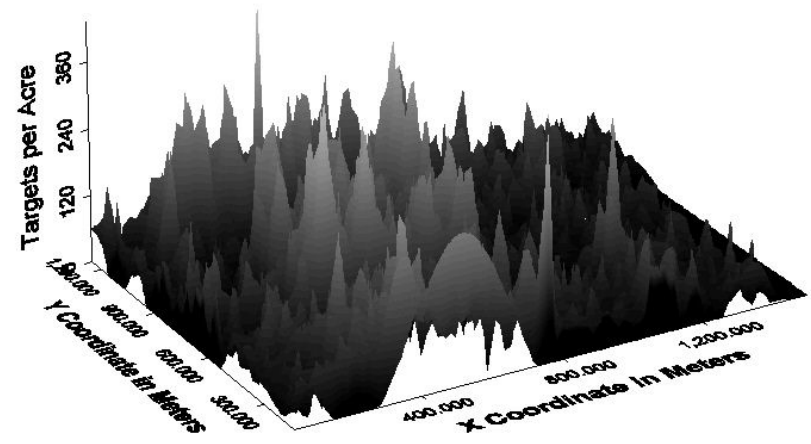

Figure 2 shows a top-down picture of Target and MEC density on the Site. The very small gray dots are the geophysicist-selected Targets. The large, darker circles show the 37 and $75 \mathrm{~mm}$ 's. Most of the small, gray circles contained Clutter. In the denser, darker regions of Figure 2, many Targets contained multiple metallic objects in the same hole. In fact, across the entire 600 acres, about $20 \%$ of all Target locations contained multiple metallic objects.

Figure 2. MEC and Target Density Map

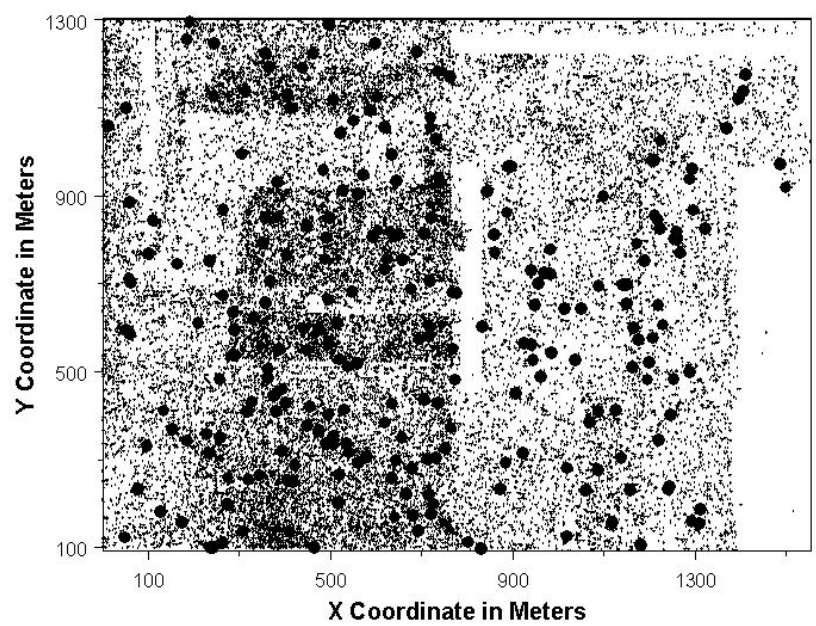

Areas containing a large number of Targets pose a particular problem for MEC discrimination because they usually contain overlapping Target DGM signatures. It is necessary to untangle such overlapping signals before they may be characterized individually. On the Site, there were 7,382 Target pairs (including $36 \mathrm{MEC}$ ) that were less than two-meters apart and 1,466 Target pairs (including $20 \mathrm{MEC}$ ) that were less than onemeter apart. Depending on the size, depth, and inclination, the DGM signatures of the Targets ranged from 0.5 to more than 3 meters in radius. Thus, overlapping Targets were a significant problem in this project.

\section{DISCRIMINATION CHALLENGES}

In addition to the overlapping Targets problem, these data posed several significant challenges in producing good discrimination results.

\subsection{Data Quality Issues}

These data were collected for comprehensive removal of all buried metallic objects on the Site. The Targets for excavation were picked by geophysicists, who examined these data using commercially available spatial-gridding and processing software, using fixed thresholds. The intent in picking Targets was to dig every Target and this was a good-quality, commercial data set for that purpose.

These data were not, however, collected for the purpose of machine-based discrimination and therefore posed significant data-quality issues for the present study. Geophysicist-driven, threshold-based Target selection normally needs only one good channel of data over any given Target to make a determination that there is likely a metallic object there. In that context, it makes no sense to incur extra costs to reacquire data when one channel has too much noise, so long as there is at least one good channel of data.

By way of contrast, our discrimination approach uses all four channels. A significant portion of the geophysicist-selected Targets had noise or calibration issues with one or two channels of data-usually channels one and/or four. We elected to retain data as long as there were at least two good channels of data and to allow the LGP algorithm to adjust for varying noise contexts. This decision permitted us to rank all 29,130 Targets for the likelihood they were MEC. However, as discussed below, it also affected the accuracy of the $37 \mathrm{~mm}$ discrimination significantly.

Figure 3. Peak Millivolt Responses for 37mm's

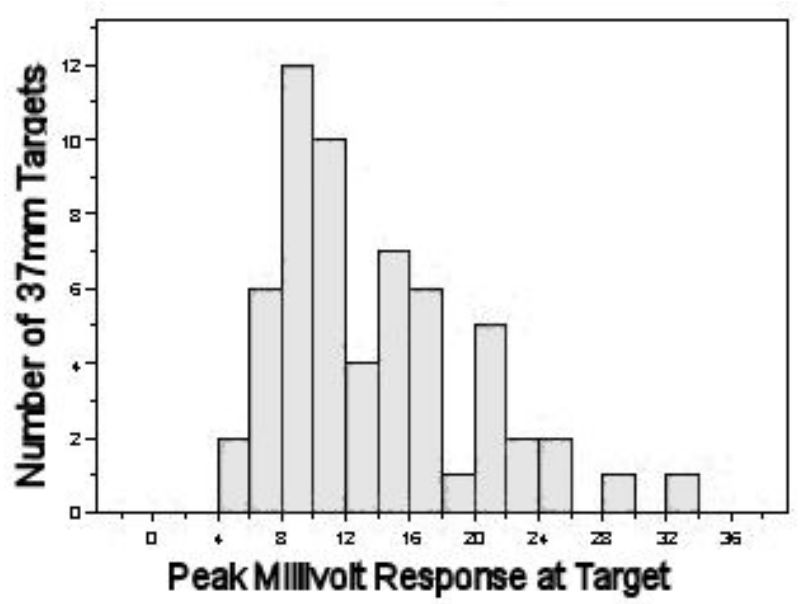

\subsection{Signal-to-Noise Ratio}

Traditional forward-modeling/inversion approaches to discrimination degrade quickly when the signal-to-noise ratio for a Target falls below 30-1. Targets in the Warren data routinely presented with a signal-to-noise ratio lower than that. The background noise level on the Site was roughly $+/-2$ to 3 Millivolts of white-noise chatter in the absence of instrument calibration or noise issues. For comparison, Figure 3 shows a 
histogram of the peak Millivolt responses for the Targets that were determined to be $37 \mathrm{~mm}$ MEC.

Using the lower figure of 2 Millivolts for the background noise level, Figure 3 shows that the largest signal-to-noise ratio for any $37 \mathrm{~mm}$ was $16-1$. And, the signal-to-noise ratio for the smaller $37 \mathrm{~mm}$ 's was just $2-1$. So the signal-to-noise ratios in these data fell below the usual 30-1 threshold.

\subsection{An Ocean of Clutter}

The most significant challenge we faced was discriminating the $37 \mathrm{~mm}$ MEC items from MEC Clutter. As noted above, $37 \mathrm{~mm}$ 's are rather small ordnance and are much more difficult to discriminate than, say, $500 \mathrm{lb}$ bombs or $155 \mathrm{~mm}$ artillery shells.

The data at Warren illustrate why this is so. There, the largest ordnance type was the $75 \mathrm{~mm}$ projectile. Investigators recovered 26,996 pieces of MEC Clutter out of the 29,130 Targets excavated. By far, MEC Clutter from detonated $75 \mathrm{~mm}$ 's was most common buried metallic item found on the site.

The dig-teams recovered only 87 intact $37 \mathrm{~mm}$ 's. They reported the intact $37 \mathrm{~mm}$ 's weighed between 2 and 20 ounces. $^{3}$ Of the MEC Clutter items recovered, 19,351 weighed between 2 and 20 ounces. Thus, most of the MEC Clutter was about the same size as the $37 \mathrm{~mm}$ 's recovered. Mass was, therefore, not an appropriate or useful discriminator for the $37 \mathrm{~mm}$ 's.

\section{BLIND-TESTING PROCEDURES}

We were provided with DGM for the entire 600 acre site. In addition, we were provided with groundtruth (that is, what the dig-teams found when they excavated a Target) for 23,085 of the 29,130 Targets, which we used to develop our models. The Targets for which we were provided groundtruth contained fiftynine, $37 \mathrm{~mm}$ 's and one-hundred eighty-six $75 \mathrm{~mm}$ 's. We were not provided groundtruth for the remaining 6,045 Targets, which contained twenty-eight $37 \mathrm{~mm}$ 's and fifty-nine $75 \mathrm{~mm}$ 's. Nor were we involved in selecting the blind Targets.

We performed two kinds of blind testing, which we distinguish here. First: In training our models to discriminate between MEC and not-MEC, we used four-fold cross-validation. All results on the 23,085 Targets on which we had groundtruth are reported on the held-out, testing data, the "virtual" blind-predictions if you will. Second: We delivered predictions to our customer for all 29,130 Targets - the Targets for which we had groundtruth and the Targets for which we did not. The predictions took the form of rankings of the Targets by the likelihood the Target was MEC. So we ranked the 6,045 Targets for which we had no groundtruth blind. After we delivered the rankings to the customer, the customer then delivered groundtruth for these 6,045 Targets to us. So, as to these 6,045 Targets, the predictions were actual blind predictions.

When we compared the blind data and the data for which we had groundtruth, it was clear that the customer had not selected 6,045 Targets entirely at random. ${ }^{4}$ Accordingly, the blind Targets were

${ }^{3}$ Weights were collected in the field by the dig-teams and were estimated manually, not weighed.

${ }^{4}$ For example, the proportion of $37 \mathrm{~mm}$ 's in the blinded data was 0.0046 . The proportion in the training data was .0025 . The not representative of the entire site. Our goal in this project was to determine what portion of Targets had to be excavated in order to clear the whole site of MEC. In order to make that assessment, it was necessary to combine predictions on the virtual blind-Targets with the predictions on the "actual" blind Targets. In that way, it was possible to fit the blind-Targets into the context of the entire site. Our reports below were assembled in this manner.

\section{EXPERIMENTAL PROCEDURE}

Our process involves several steps, described below. Before LGP was applied, considerable preprocessing to remove noise and standardize the Targets was necessary. In addition, for each Target we had to define what data points were in the Target and which were not - that is, we had to define the size, shape, and orientation of each Target. Finally, we extracted the features used for LGP discrimination, analyzed them and prepared preliminary statistical models from them. The process is described in more detail below.

\subsection{Preprocessing}

EM61 data contains low frequency variation caused by factors like instrument drift or geomagnetic variation. Targets are typically between 0.5 and 3 meters in radius. We filtered the low frequency variation using a robust, high-pass filter, allowing features less than 10 meters in length to pass. This filtering process was entirely automated--the volume of data prevented individual examination of Targets. Then we standardized the background noise regions to a mean level of about zero.

Figure 4. Channel 1 (dark line), Channel 2 (middle line), and Channel 3 (bottom line) on a Transect before Preprocessing

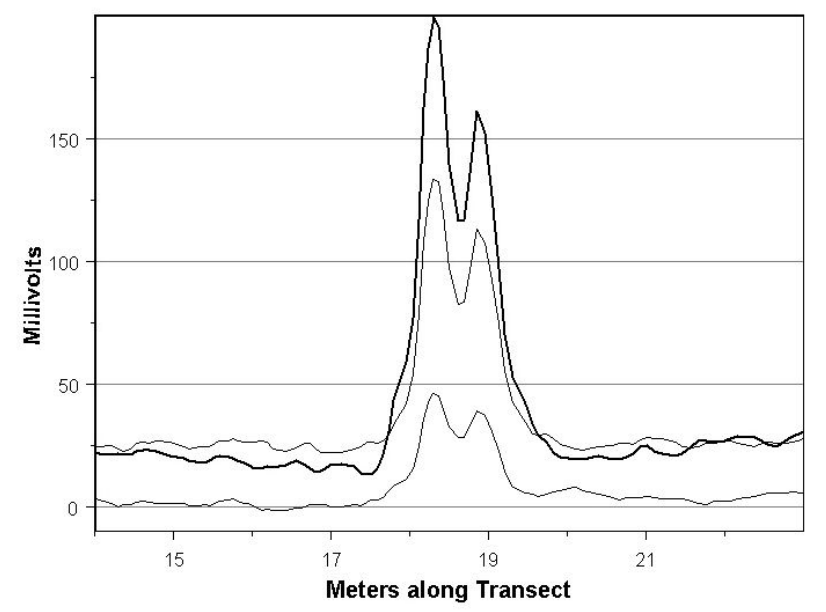

Figure 4 shows Channels 1, 2 and 3 of an EM61 MK2 before we preprocessed the data. It shows about ten meters of data from a single transect in the vicinity of a Target. Note the differences between channels in background noise levels and the eleven Millivolt rise in channel one from meter 20 to $23 .^{5}$ Figure 5 shows

probability of this happening by random chance is less than $\mathrm{p}=0.007$. In addition, the blinded data had a disproportionately high percentage of $37 \mathrm{~mm}$ targets where Channel 1 was excessively noisy over the target.

5 The eleven Millivolt rise in Figure 4 looks small in comparison to the Target shown therein. But Figure 3 reveals that almost 
the same portion of that transect after preprocessing. The background levels for all three channels have been standardized to approximately zero and much of the eleven Millivolt rise has been eliminated.

Figure 5. Channel 1 (top, dark line), Channel 2 (middle line), and Channel 3 (lowest line) on a Transect after Preprocessing

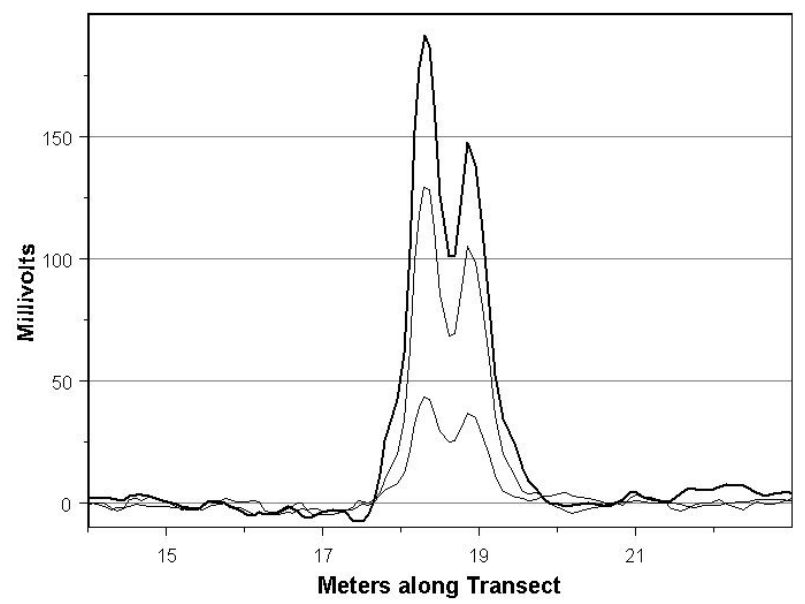

\subsection{Defining the Targets}

The customer provided us $\mathrm{x}, \mathrm{y}$ coordinates of the geophysicistselected Targets. We grouped the EM61 signals read in the vicinity of each Target as being: (1) in the Target; and/or (2) in another (adjacent) Target; or (3) in the background noise. Our process for doing so involved fitting an ellipse to each Target using the preprocessed data converted to localized z-scores. The localized z-scores were calculated for each Target empirically, relative to the background noise level in the ten meter circle surrounding the Target.

We used a deterministic optimizer using the Lipchitz global optimization algorithm [17] to define the Targets from the localized z-scores. The problem posed to the optimizer was to find the ellipse that best separated the above-background-noise values $(z$-score $>2)$ in the vicinity of each Target from the belowbackground-noise values $(z$ score $<=2)$ in the vicinity of that Target. For overlapping Targets, points in the area of overlap were excluded from the optimization. Thereafter, the ellipse derived was used to define the size and shape of the Target. We defined such an ellipse for each channel of data for each of the 29,130 Targets. This process was also entirely automated.

Figure 6 shows the result of this process for a single EM61 channel around one Target. Each datum from the EM61 near the geophysicist-picked Target location (marked with an "X") is shown as a point. The size of the points in Figure 6 represents a point's z-score-larger means a higher z-score relative to background noise. The ellipse defines which points we treated as being "in" the Target.

half of the $37 \mathrm{~mm}$ 's had a peak response above background noise that was less than or equal to eleven millivolts. So removing low frequency movements in the signal like the one shown in Figure 4 is quite important.

\subsection{Feature Extraction}

Once the ellipse was defined, we extracted a series of features for each Target. We determined what features to extract using two criteria. First: approximate physics-based models of induced magnetic field provides some guidance as to what ought to be important. See e.g. [4][3]. For example: (1) The shape of the timedecay of the signal from Channel 1 to Channel 3 shows different characteristics for differently shaped objects; (2) The ratio of the upper and lower coil signals ought to provide information about the depth of the object because of the exponential decay of signal strength with distance; and (3) The symmetry of the signal about the major and minor axes is affected by the shape and inclination of the buried object. So the features we extracted provided detailed information about decay, upper/lower coil ratios, and signal symmetry.

Figure 6. Ellipse defining a Target

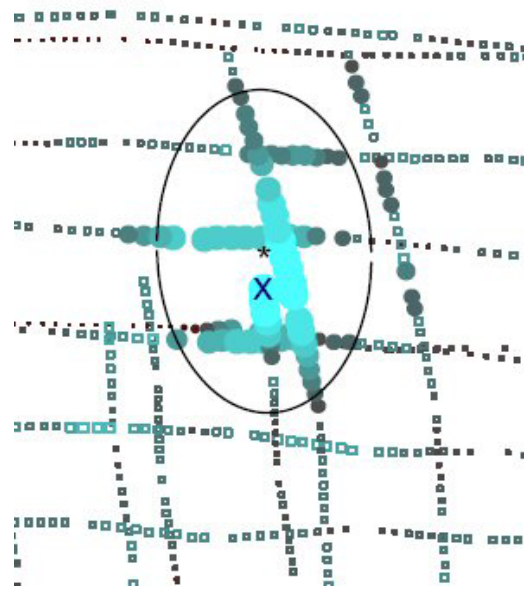

But approximate physics-based models do not completely define the problem posed by production-grade survey data. Instrument noise of various types affects the signal. For example, we described situations above where one or more channels would be overly noisy. The physics of induced magnetism cannot account for such problems; yet models must be built from imperfect data.

To account for these imperfections of the data, we also extracted features that provide a detailed statistical topology of each Target. For example, we extracted the median signal value for the innermost part of each ellipse and for concentric ellipsoidal donuts outside that innermost part.

\subsection{Feature Reduction}

We took the extracted feature set and subjected them to statistical correlation analysis to determine which were most predictive of "MEC-ness" and, of those, which features were the least correlated with each other. In addition, we performed a series of preliminary modeling runs to determine which features contributed most to good-quality models [10]. After three iterations of this process, we had a tractable-size feature set.

\subsection{Modeling}

We then built our models using four-fold cross-validation - that is, the data set was split four different ways, with each data point serving as training data three times and as blind, testing data once. Each split of the data set was generated randomly. The examples 
of MEC were duplicated until there was an approximate balance between the number of MEC items and the number of non-MEC items in each cross-validation training set. No such duplication was performed for the data used to test the models.

Our principal tool here was LGP [2][10][16]. That software performs multiple runs automatically and selects the best evolved programs out of multiple runs. We used the default parameters of the LGP software for in non-stepping mode [10] and terminated each run when no improvement had occurred for at least 50 runs.

The Target output we wanted to predict was Boolean - a value of 1 represented MEC and a value of 0 represented non-MEC. The output from our models was a probability that an item was MEC.

\section{Figure 7. ROC Chart for $75 \mathrm{~mm} \mathrm{MEC}$}

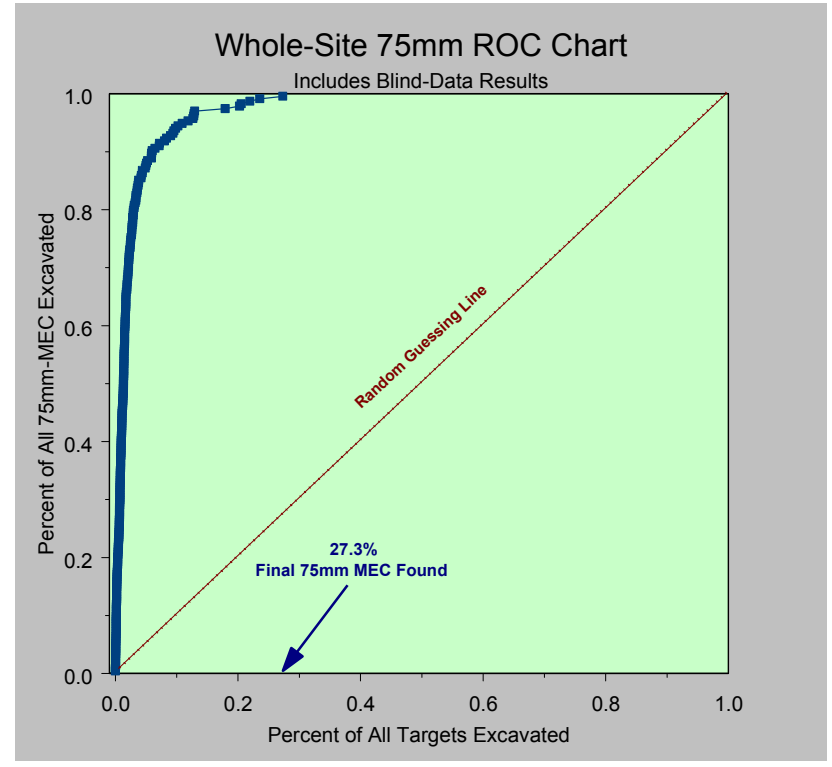

For each of the cross-validation data sets, we chose the model that our software selected as best on the training data. The numbers reported below represent the performance of the four chosen models on the held-out, testing data - the "virtual" blind data.

For our predictions on the "actual" blind Targets, we took the four models trained on the four cross-validation data sets, applied them to the features extracted for the "actual" blind Targets, and averaged the predictions from the four models. That average was the value used to order the blind Targets.

We performed this process separately for $37 \mathrm{~mm}$ and $75 \mathrm{~mm}$. Using the outputs of the four models described above on both the "virtual" blind Targets and the "actual" blind Targets, we prepared a list prioritizing the Targets starting with the most likely to be MEC to the least likely to be MEC. We did that on a "whole site" basis to show where the "actual" blind Targets would have been excavated as part of clearing the entire site.

\section{RESULTS}

\subsection{5mm MEC Results}

Figure 7 is a pseudo-Receiver Operating Characteristics ("ROC") chart [13] showing the performance of our prioritized dig-list on $75 \mathrm{~mm}$ MEC. The horizontal axis represents the prioritized diglist-that is, what portion of all Targets would have been excavated had the site been cleared in the order specified by our prioritized dig-list. The vertical axis shows what portion of the $75 \mathrm{~mm}$ 's would have been cleared had the site been cleared in the order suggested by our prioritized dig-list. The diagonal line represents what random guessing would generate. The line with square points is key. It shows the tradeoff between finding all $75 \mathrm{~mm}$ 's, on the one hand, and the number of Targets that must be dug to do so, on the other hand, given our prioritized dig list.

The horizontal axis in Figure 7 is somewhat different than what would be shown in a true ROC curve. In a true ROC curve, the horizontal axis would show the portion of true-negatives excavated in the order of the prioritized dig-list [13]. We use the pseudo-ROC chart for display as that is the recommended DoD format for assessing rankings to MEC vs. not-MEC in remediation discrimination [5].

In brief summary, Figure 7 shows that all $75 \mathrm{~mm}$ MEC would have been cleared from the site by excavating only $27.8 \%$ of the total Targets had our dig-list been used to order the excavation. Put another way, our approach would have cleared all $75 \mathrm{~mm}$ 's and reduced the number of false-alarms to do so by $72.2 \%$.

\section{Figure 8. ROC Chart for $37 \mathrm{~mm}$ MEC}

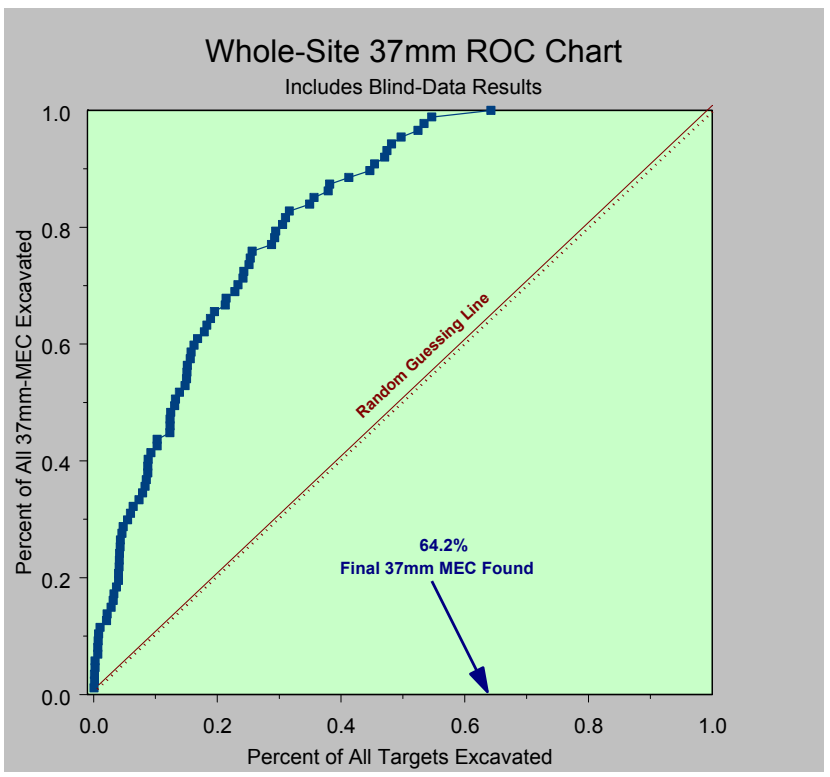

Our test of the statistical significance of these rankings begins by converting the pseudo-ROC curve in Figure 7 into a true ROC curve, described above. Of course, the true ROC curve for these rankings also has a 45 degree random-guessing line that goes thru origin and the point 1,1 . The area under that random guessing line is, obviously, 0.5. So our null hypothesis is that the area under the true ROC curve for our $75 \mathrm{~mm}$ rankings is not greater than 0.5 . The alternative hypothesis is that the area under the ROC curve is greater than 0.5 .

The computation of the area under a ROC curve and the standard error of the area are described by Hanley in [13]. For our $75 \mathrm{~mm}$ rankings, the area under the ROC curve is 0.9568 and the standard error is 0.00918 . Thus, 0.5 is more than 49 standard deviations away from the computed area under the ROC curve. The p-value that the area under this ROC curve was not greater than 0.5 is zero to the limits of computational precision. Accordingly, we 
reject the null hypothesis for the $75 \mathrm{~mm}$ rankings with a high level of confidence.

\subsection{7mm MEC Results}

Figure 8 is a pseudo-ROC chart showing the performance of our prioritized dig-list on $37 \mathrm{~mm}$ MEC. The horizontal axis represents the prioritized dig-list. The vertical axis shows what portion of the $37 \mathrm{~mm}$ 's would have been cleared had the site been excavated in the order suggested by the prioritized dig-list. The diagonal line again represents what random guessing would generate.

In summary, Figure 8 shows that all $37 \mathrm{~mm}$ MEC would have been cleared from the site by excavating only $64.2 \%$ of the total Targets. Put another way, our approach would clear all $37 \mathrm{~mm}$ 's and reduce the number of false alarms by $35.8 \%$.

We applied the same Hanley procedure [13] used for the $75 \mathrm{~mm}$ ROC curve to test the statistical significance of our $37 \mathrm{~mm}$ rankings. The results were: (1) The area under the true-ROC curve for the $37 \mathrm{~mm}$ rankings is 0.8233 ; (2) the standard error of the area is 0.0275 ; and (3) The probability that the area under the $37 \mathrm{~mm}$ true-ROC curve is not greater than 0.5 (random guessing) is $3.27 \mathrm{E}-32$ to the limits of machine precision. Accordingly, we reject the null hypothesis with a high degree of confidence.

\section{DISCUSSION}

These results represent a significant step forward in the state-ofthe-art for MEC discrimination in several regards.

First, this study extends the approach described by Francone and Deschaine in [12] from small, high-quality data samples derived from a simulated impact site to noisy, production-grade, survey data. To our knowledge, this is by far the largest MEC discrimination test reported and the only one demonstrating substantial success on production-grade, survey data.

Second, this study replicates Francone's findings in [12] on a much larger data set in blind, third-party-conducted testing. Both [12] and the present study reduced false alarms by between $35 \%$ and $40 \%$ over geophysicist Target-selection in clearing a site of the smallest ordnance. (In [12], the smallest ordnance was $20 \mathrm{~mm}$ MEC. In the present study, the smallest was $37 \mathrm{~mm}$.) Both this study and Francone's previous work used LGP. Both used similar methodologies. Thus, the replication of these earlier results on blind-data provides substantial evidence for the efficacy of the methodology.

Third, these results improve on results for larger ordnance reported by well conducted studies using the forwardmodeling/inversion approach we described earlier. Nelson conducted a relatively large study of an actual impact site using magnetic and EM61 survey data collected by the MTADS system [9]. MTADS produces much higher quality survey data than we had access to in this project. ${ }^{6}$ The discrimination approach tested by Nelson produced a $58 \%$ reduction in false alarms for $81 \mathrm{~mm}$ mortars, when the $81 \mathrm{~mm}$ 's were considered alone. (In general, the larger the ordnance, the easier it is to discriminate. So our

\footnotetext{
${ }^{6}$ This difference in data-quality is not a result of different datagathering technique. Rather, it is the result of different hardware platforms. MTAD's is the current state-of-the-art for surveybased data collection but is seldom used for actual cleanup because of expense. [8]
}

discrimination of $75 \mathrm{~mm}$ projectiles in the present study was a roughly comparable task to the discrimination of $81 \mathrm{~mm}$ 's in [9].) We produced a $72.2 \%$ reduction in the false alarms for $75 \mathrm{~mm}$ 's on data very much inferior to that group's MTAD's data.

Fourth, the discrimination of small ordnance (such as $37 \mathrm{~mm}$ 's) from Clutter is usually the most difficult discrimination task on a site. We are not aware of any studies that have produced results on small ordnance using survey-based, production-grade data that show results comparable to ours, other than Francone's previous work also using LGP and similar preprocessing methodologies to the present study and even that was conducted on a much smaller data set [12].

Fifth, we mentioned earlier that we encountered noise problems with the signal on channels one and/or four from the EM61 MK2. This problem affected 28 of the $37 \mathrm{~mm}$ 's on the site. This was a problem for discrimination as it affected various measurements of the shape of the decay curve across the three time gates for these Targets, all important discriminators. For these Targets, the models we developed solved the discrimination problem differently than they solved the same problem for the less noisy Targets. Had we not encountered these noisy data, the entire site would have been cleared of $37 \mathrm{~mm}$ 's by about $50 \%$ of the way through our prioritized dig-list. That is, if the twenty-eight $37 \mathrm{~mm}$ 's that had noise problems in channel one been removed from the study, our results would have improved to $50 \%$ from $64.2 \%$. There are two conclusions to draw from these observations: (1) Better data means better discrimination; and (2) Our methodology permits our models to adapt to noise issues on a site. Thus, where the data for a particular Target was not especially good, an acceptable solution (albeit not as good as the solutions for clean-data Targets) was, nevertheless, derived, which provided good discrimination, even in the face of noise.

All this may suggest why the LGP approach has produced better results than previous forward modeling/inversion studies. At their core, both approaches use the known physics of induced magnetic fields-one explicitly via the forward model and the other implicitly via the extracted features provided to LGP. But known physics is not all that is taking place in real production projects. Like many other earth-science challenges, a comprehensive closed-form analytic solution for MEC discrimination is not available, only approximations. In addition, instruments drift and go out of calibration. Positioning is not exact. The geophysicists do not walk straight lines exactly one-meter apart across the site and transects are not always directly over the Target. In other words, there is imprecision in the data that comes and goes. LGP can model how the noise on a site affects the signal, something that the purely physics based approaches cannot easily do.

Finally, the forward modeling approach reduces all information about a Target to just a few parameters - usually between two and five. We examined the features drawn from the DGM that proved to be useful discriminators in our models and subjected them to principal components analysis ("PCA"). PCA produces uncorrelated components that contain most of the variance in the original data set [14]. More than 25 of these orthogonal components contained useful and statistically significant information for distinguishing MEC from not-MEC. This suggests that there is some information loss in reducing the description of a Target using a lumped parameter formulation comprising of only two to five orthogonal parameters. 
By allowing LGP to fuse the known physics and extract valuable discrimination information from what might appear as noise characteristics of the instrument, the data gathering techniques, or the signal itself, and by not requiring that models be restricted to a very small number of parameters to describe a Target, we believe it possible to extend MEC discrimination accuracy beyond what may be had by relying solely on the analytical physics-based, forward-model approximations.

\section{ACKNOWLEDGMENTS}

Our thanks to John Wright, Chief of Restoration at Warren Air Force Base and to Brian Powers and Andy Gascho of URS Corp. for making this project possible. We also express our gratitude to C. Edward Dilkes and Robert "Tom" Weatherly, without whose direction and vision, MECFinder would not have been developed.

\section{REFERENCES}

[1] Banks, R. E., Núñez, E., Agarwal, P., Owens, C., McBride, M., and Liedel, R., 2005. Genetic Programming for Discrimination of Buried Unexploded Ordnance (UXO)). Late-breaking paper at The Genetic and Evolutionary Computation Conference (GECCO-2005)

[2] Banzhaf, W., Nordin, P. Keller, R. Francone, F. Genetic Programming, an Introduction, Morgan Kaufman Publishers, Inc., San Francisco, CA (1998).

[3] Billings, L.R., and Oldenburg, D. W. (2003). Joint and Cooperative Inversion of Magnetic and Time Domain Electromagnetic Data for the Characterization of UXO, Proceedings of the Symposium on Application of Geophysics to Environmental and Engineering Problems 2003 (CD), Environmental and Engineering Geophysical Society, San Antonio, TX, 2003.

[4] Billings, S. D., Pasion, L. R., and Oldenburg, D. W. (2003). Discrimination and Classification of UXO Using Magnetometry: Inversion and Error Analysis Using Robust Statistics, Proceedings of the Symposium on Application of Geophysics to Environmental and Engineering Problems 2003 (CD), E.E.G.S., San Antonio, TX, 2003.

[5] Cespedes, E.: Advanced UXO Detection/Discrimination Technology Demonstration-U.S. Army Jefferson Proving Ground, Madison, Indiana. US ACOE, Engineer Research and Development Center, ERDC/EL TR-01-20 (2001).

[6] Defense Science Board Task Force. Report of the Defense Science Board Task Force on Unexploded Ordnance. Department of Defense. December (2003).

[7] Deschaine, L. M., Hoover, R. A., Skibinski, J. N., Patel, J. J., Francone, F. D., Nordin, P. and Ades, M. J.: Using Machine Learning to Compliment and Extend the Accuracy of UXO
Discrimination Beyond the Best Reported Results of the Jefferson Proving Ground Technology Demonstration. In: Proceedings of the Society for Modeling and Simulation International's Advanced Technology Simulation Conference, April 2002. San Diego, CA, USA (2002).

[8] Environmental Security Technology Certification Program. Multi-Sensor Towed Array Detection System, Cost and Performance Report (UX-9526). September 1999.

[9] Environmental Security Technology Certification Program. Environmental Induction and Magnetic Sensor Fusion for Enhanced UXO Target Classification, Cost and Performance Report (UX-9812). February 2004.

[10] Francone, F. D., Discipulus Owner's Manual. RML Technologies, Inc. (2002). Available at www.aimlearning.com.

[11] Francone, F. D., and Deschaine, L.M., Extending the Boundaries of Design Optimization by Integrating Fast Optimization Techniques with Machine-Code-Based Linear Genetic Programming, Information Sciences JournalInformatics and Computer Science, Elsevier Press, Vol. 161/3-4 pp 99-120: 2004. Amsterdam, the Netherlands.

[12] Francone, F. D., Deschaine, L. M., Battenhouse, T., and Warren, J. J., 2004. Discrimination of Unexploded Ordnance from Clutter Using Linear Genetic Programming, Proceedings of the Genetic and Evolutionary Computation Conference, Late Breaking Papers, 2004, Seattle, WA, USA.

[13] Hanley, J. and McNeil, B., The Meaning and Use of the Area under a Receiver Operator Characteristic (ROC) Curve," Radiology, Vol. 143, pp. 29-36, 1982.

[14] Jolliffe, I.T. Principal Components Analysis, Second Edition. (Springer Series in Statistics, NY, NY 2002), p. 1.

[15] Nelson, H. H., Altshuler, T., Rosen, E., McDonald, J.R., Barrow, B., and Khadr, N. Magnetic Modeling of UXO and UXO-Like Targets and Comparison with Signatures Measured by MTADS, www.citeseer.ist.psu.edu/273329.html

[16] Nordin, P., Francone, F. Banzhaf, W. Efficient Evolution of Machine Code for CISC Architectures Using Blocks And Homologous Crossover. In: Advances in Genetic Programming 3, MIT Press, Cambridge, MA (1998).

[17] Pinter, J., Global Optimization in Action. Continuous and Lipschitz Optimization: Algorithms, Implementations and Applications. Kluwer Academic Publishers, Dordrecht / Boston / London, 1996.

[18] Tantum, S., Yu, Y., Zhu, Q., Wang, Y., and Collins, L. http://www.ee.duke.edu/research/collins/uxodocs.html, March 28, 2006 